ACTA UNIVERSITATIS LODZIENSIS

Folia Litteraria Romanica 15, 2020

https://doi.org/10.18778/1505-9065.15.08

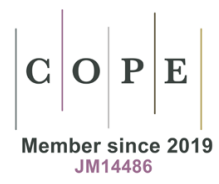

\author{
Magdalena Koźluk \\ Université de Lodz \\ (iD) ORCID ID : 0000-0001-7775-3594 \\ magdalena.kozluk@uni.lodz.pl
}

\title{
Digression sur le « mespris » dans les Erreurs populaires de Gaspard Bachot
}

\begin{abstract}
RÉSUMÉ
Dans le discours scientifique au XVII ${ }^{\mathrm{e}}$ siècle en France, la digression est un procédé rhétorique souvent utilisé par les auteurs. Une excursion longue de onze pages reste cependant une rareté dans l'art d'Esculape, plus encore quand elle traite du mépris. Dans cet article, nous nous proposons d'analyser une digression sur les causes du mépris du milieu médical se trouvant dans les Erreurs populaires de Gaspard Bachot (1570-1630), conseiller et médecin du Roy à Moulin. Afin de faciliter la compréhension du mépris et de son objet dans le contexte du savoir médical, nous essayons d'abord de situer ce fragment dans le cadre de la genèse de l'ouvrage lui-même (Les Erreurs populaires de Bachot comme continuation des Erreurs populaires de Laurent Joubert). Ensuite, nous étudions le contenu de la digression bachotienne afin d'en dégager les principaux motifs du mépris relevés par le médecin dans la médecine de l'époque. Leur analyse fera ensuite apparaître une hiérarchie du corps médical. Nous terminerons notre parcours par la présentation d'un manifeste éthique embrassant le canon des règles qui, seules, selon Bachot, peuvent sauver la médecine.
\end{abstract}

MOTS-CLÉS - médecine au XVII ${ }^{\mathrm{e}}$ siècle, Erreurs populaires, Laurent Joubert, Gaspard Bachot, digression, mépris, causes, abus, ignorance, conventions

\section{"Digression on 'Contempt' in Gaspard Bachot's Medical Work Les Erreurs populaires"}

\section{SUMMARY}

Digressions are not uncommon in medical treatises published in France during the Seventeenth century, but rare are those of more than a few pages in length, and rarer those that treat of «le mespris ». The digression we consider here is found in Les Erreurs populaires, by Gaspard Bachot (1570-1630), a bourbonnnais physician, conseiller et médecin du Roy, in Moulin. We first situate this digression which takes up eleven pages of Bachot's book, in the context of the medical knowledge of the period, and trace its genre and its genesis: Bachot's Erreurs were written as a continuation of the Montpellier Professor of Medicine Laurent Joubert's own Les Erreurs populaires. We then show the reasons of the contempt shown, during the period, in and for medicine, as an academic physician like Bachot sees them. Finally, his balanced view of the established hierarchy of the medical professions, leads 
him to propose a set of Commandments that need to be honoured by young physicians, in order to ensure a professional ethical conduct.

KEYWORDS - medicine XVII ${ }^{\text {th }}$ century, Erreurs populaires, Laurent Joubert, Gaspard Bachot, digression, contempt, causes, abuses, ignorance, conventions

« Ceste digression a esté plus longue que je ne pensois » ${ }^{1}$, écrit Gaspard Bachot (1570-1630), «Conseiller et Medecin du Roy à Moulin » ${ }^{2}$, avant d'ajouter : « mais combattant les Erreurs de la Medecine et son mespris d'aujourd'huy, où elle va en decadence, si on ne la releve, je voudrais en ces erreurs contribuer mon talent à desraciner cest erreur $»{ }^{3}$. Si, dans le discours scientifique de l'époque, la digression n'est pas en soi un procédé rhétorique exceptionnel ${ }^{4}$, $1^{\prime}$ historien trouvera dans ce développement sur le mépris de la médecine, long de onze pages, une intervention d'autant plus remarquable qu'elle est rare sur ce sujet dans le discours médical du XVII ${ }^{e}$ siècle. Nous souhaitons nous pencher sur le passage mentionné car il apporte, selon nous, un éclairage précieux sur les causes de la contemptio medicinae alléguées par notre médecin universitaire. Pour faciliter la compréhension du mépris, et surtout de son objet dans le contexte du savoir médical, nous tâcherons d'abord de situer cette excursion dans le cadre génétique de l'ouvrage lui-même : Les Erreurs populaires de Laurent Joubert ${ }^{5}$ vs Les Erreurs populaires de Gaspard Bachot. Ensuite, nous examinerons en détail le contenu de la digression de Bachot afin d'en

1 G. Bachot, Erreurs populaires touchant la médecine et régime de Santé. Euvre nouvelle, desirée de plusieurs, et promise par feu M. Laurens Joubert, Lyon, Barthelemy Vincent, 1626, p. 174. Notons que l'édition princeps des Erreurs populaires fut publiée en 1626 chez Barthélemy Vincent (153 ?-159 ?), imprimeur protestant. C'est le 17 septembre 1625 que Gaspard Bachot lui confie l'impression de son ouvrage, ayant obtenu de Louis XIII le privilège pour dix ans. Toutefois, il est intéressant de noter que Barthélemy Vincent permet la même année à la veuve de Thomas Soubron, Philiberte, de faire sortir des presses de son défunt mari les Erreurs populaires de Bachot, mais sous un autre titre (Partie troisiesme des Erreurs populaires : touchant la médecine et régime de santé, en suitte de celles de feu M. Laurens Joubert, contenant cinq livres. Euvre nouvelle, desirée de plusieurs, et promise par ledit feu Joubert, Lyon, pour la veuve de feu Thomas Soubron, 1626) et avec une nouvelle page de titre, arrangée par la veuve elle-même.

2 G. Bachot, Erreurs populaires touchant la médecine et régime de Santé. Euvre nouvelle, desirée de plusieurs, et promise par feu M. Laurens Joubert, Lyon, Barthelemy Vincent, 1626, page de titre.

3 Ibid., p. 174.

4 G. M. Poutingon, Poétique du digressif. La digression dans la littérature de la Renaissance, Paris, Classiques Garnier, collection "Études et essais sur la Renaissance », 2012 ; Anecdotes philosophiques et théologiques de l'Antiquité aux Lumières, éd. F. Lecercle, G. Navaud, Paris, Classiques Garnier, 2012, p. 7-18 ; Dans le discours préfaciel de l'époque, les commentaires évoquant le mépris de la médecine et «son anéantissement », ont déjà acquis le statut de véritables topoï ; cf. M. Koźluk, L'Esculape et son art à la Renaissance. Le discours préfaciel dans les ouvrages français de médecine 1528-1628, Paris, Éditions Classiques Garnier, 2012, p. 66-75.

5 L. Joubert, Erreurs populaires au fait de la medecine et regime de santé, Bordeaux, Simon Millanges, 1578. 
dégager les principaux motifs du mépris de l'art d'Esculape. Leur inventaire et leur analyse nous conduiront ensuite à restituer une image nuancée du corps médical de cette époque. Nous terminerons enfin par la présentation d'un manifeste éthique embrassant le canon des règles qui, seules, selon lui, pourront sauver la médecine et « repurger l'auge qui salit une si excellente profession $»^{6}$.

\section{1. « Ne pas mespriser, ains remonstrer»}

Commençons par la figure de Gaspard Bachot, auteur de la digression qui nous intéresse. Dans l'histoire de la médecine du XVII ${ }^{e}$ siècle, son nom est connu pour deux raisons. En premier lieu, il a témoigné d'un grand dévouement intellectuel envers Laurent Joubert (1529-1583), professeur de médecine et chancelier de l'université de Montpellier ${ }^{7}$ qui, ayant $1^{\prime}$ ' esprit orné de beaucoup de connaissances $»^{8}$, n'a pas manqué de se dégager " des préjugés de son siècle »", en composant plusieurs livres importants. Certains d'entre eux furent immédiatement appréciés, d'autres, en revanche, donnèrent lieu à toutes sortes de controverses ${ }^{10}$. En second lieu, Gaspard Bachot reste l'auteur d'un unique ouvrage dont l'ambition fut de poursuivre l'un des travaux majeurs inaugurés jadis par Joubert, Les Erreurs populaires. Il en résulte que la troisième partie des Erreurs populaires que propose Bachot, « desirée de plusieurs, et promise par ledit feu Joubert ${ }^{11}$, s'avère être le fidèle prolongement de l'œuvre de son maître à penser ${ }^{12}$.

6 G. Bachot, op. cit., p. 162.

7 Sur la vie et l'œuvre du médecin, voir L. Dulieu, La Médecine à Montpellier, Avignon, Presses Universelles, t. II, La Renaissance, 1979, p. 340-343. Voir aussi M. Tiollais, La Médecine et le régime de santé. Des erreurs populaires et propos vulgaires, $t$. I, Livre I : réfuté et expliqué par Laurent Joubert, Paris, Harmattan, 1997, p. 23-32.

8 J.-F. Michaud, Biographie universelle, Paris, Delagrave, 1843-1865, t. 21, p. 222.

9 Ibid.

${ }^{10}$ Pour les controverses voir à titre d'exemple : L. Joubert, Traité $d u$ ris, Paris, Nicolas Chesneau, 1579 ; ou encore L. Joubert, Annotations sur toute la Chirurgie de M. Guy de Chauliac, Tournon, Claude Michel, 1598. Voir aussi G. de Rocker, «Le rire au temps de la Renaissance : Le Traité du ris de Laurent Joubert », Revue Belge de Philologie et d'Histoire, 1978, 56-3, p. 629-640 ; M. Lazard, « La Thérapeutique par le rire dans la médecine du XVI e siècle » in Littérature et Pathologie, dir. M. Milner, Saint-Denis, Presses Universitaires de Vincennes, 1989, p. 13-27 ; R. Gorris Camos, «Penser le rire et rire de cœur : le Traité du ris de Laurent Joubert, médecin de l'âme et du cœur » in Rire à la Renaissance, édit. M.-M. Fontaine, Genève, Droz, 2010, p. 156-157.

${ }^{11}$ G. Bachot, op. cit., page de titre.

${ }^{12}$ Bachot formule dès le début les objectifs de son livre : «Et puis si je blasme ceux qui souillent si noble vacation que la nostre, ne suis-je pas excusable, puis qu'en la suitte de ces Erreurs que je poursuy, je desire deraciner l'erreur qui s'y commet? m'asseurant que feu Monsieur Joubert à qui estoit deub l'achevement de cest œuvre comme le commancement, par son auctorité et sa doctrine les eust mieux vannez que moy, à qui l'un et l'autre defaut. Esperant neantmoins que ses heureuses ombres auront nostre tache aggreable, nous remuerons notre tonneau Diogenique pour en repurger l'auge qui salit une si excellente profession », G. Bachot, op. cit., p. 162. 
Rappelons que Les Erreurs populaires ${ }^{13}$ de Joubert avaient pour dessein de « tancer l'imbécillité populaire [...] montrant toujours l'infaillible supériorité du savoir médical sur la superstition $»^{14}$. En dédiant en 1578 ce livre singulier à Marguerite de Valois ${ }^{15}$, véritable « deesse $»^{16}$ dont l'esprit fut déclaré « plus net que la perle, tres-admirable an capacité, vivacité, dexterité, et solidité, speculation, perquisicion, et explication des choses les plus ardues et difficiles $\gg{ }^{17}$, Laurent Joubert soulignait les deux buts principaux de son entreprise : le premier était d' « instituer la jeunesse an ditte sciance, tant par ecrit, que doctrine verbale, sinceremant et dilligemment, luy donnant les premiers trais, l'abreuvant de bon preceptes, l'elevant aus plus secrets remedes, l'exerçant an dispute et an pratique $\|^{18}$; le deuxième cherchait à « etaindre et aneantir plusieurs fausses opinions, et les erreurs (angeance d'ignorance) qui ont longuemant eu valeur et vogue an la medecine, chirurgie et apoticairie $»^{19}$. Fidèle au savoir dogmatique et rationnel de l'université, Joubert pressentait que ces opinions, « tant epaisses, grossieres et lourdes $»^{20}$, risquaient de nuire à la santé des gens et de mener à nombre d'abus.

Plutôt que de les «mespriser ou dissimuler », le docte médecin trouva plus conforme à l'enseignement humaniste de " remontrer au vulgaire ignorant, an quoy et comment il s'abuse et forvoye, le remettant an un melheur chemin $»^{21}$. On jugera cette attitude d'autant plus estimable qu'elle fut celle d'un professeur de médecine, de surcroît, Chancelier de la célèbre Université de Montpellier, qui se montre préoccupé de « dissuader les fausses opinions du vulgaire » ${ }^{22}$, de «l'instruire de faire mieus ce que luy concerne $»^{23}$.

${ }^{13}$ Sur ce genre littéraire, nous renvoyons à l'étude de J. Coste, La Littérature des 'erreurs populaires': une ethnographie médicale à l'époque moderne, Paris, Champion, 2002.

${ }^{14}$ X. de Saint-Aignan, «Les Songes de Phestion de Pierre Bailly », in "Vulgariser la médecine : du style médical en France et en Italie (XVI ${ }^{e}$ et XVII ${ }^{\mathrm{e}}$ siècles) », éd. A. Carlino, M. Jeanneret, Genève, Droz, 2009, p. 141.

${ }^{15}$ Sur le choix du dédicataire, Marguerite de Valois, ainsi que sur les circonstances de ce choix, voir A. Gilles-Chikhaoui « Marguerite de Valois, dédicataire des Erreurs populaires de Laurent Joubert : un patronage audacieux ?» [En ligne], 1|2019, mis en ligne le 22 juin 2019, URL http://www.siefar.org, consulté le 16.07.2019. Sur la tradition humaniste des femmes en tant que dédicataires voir É. BerriotSalvadore, Les Femmes dans la société française de la Renaissance, Genève, Droz, 1990, p. 369-390; S. Broomhall, «Au-delà de la cour : patronnes et mécènes du manuscrit à l'imprimé », in Patronnes et mécènes en France à la Renaissance, K. Wilson-Chevalier dir., Saint-Étienne, Publications de l'Université de Saint-Étienne, 2007, p. 45-58; Cl. Martin-Ulrich, La Persona de la princesse au XVI siècle : personnage littéraire et personnage, politique, Paris, H. Champion, 2004.

${ }^{16}$ L. Joubert, Erreurs populaires, op. cit., fo a $8 \mathrm{v}^{0}$.

${ }^{17}$ Ibid.

${ }^{18}$ Ibid., fo a $5 \mathrm{r}^{\circ}$.

${ }^{19}$ Ibid., $\mathrm{f}^{\mathrm{o}}$ a $5 \mathrm{v}^{\mathrm{o}}$.

${ }^{20}$ Ibid.

${ }^{21}$ Ibid.

${ }^{22}$ Ibid.

${ }^{23}$ Ibid., f $\mathrm{f}^{\mathrm{o}} 6 \mathrm{r}^{\mathrm{0}}$. 
La mission que Joubert se donne ne sera cependant pas privée de difficultés majeures, mais c'est avec beaucoup de naturel qu'il semble en accepter la charge. Il y voit d'abord le « devoir du médecin » qui résulte du rang supérieur de l'ars medica parmi toutes les sciences, il entrevoit ensuite comment le rôle de l'instituteur peut s'inscrire dans le canon des règles et donner du prestige à son èthos de médecin ${ }^{24}$. En somme, bien que le terme " mépris » n'apparaisse que rarement dans le texte de Laurent Joubert, le lecteur comprend qu'adressé au « peuple ignorant $»^{25}$ ou à l' " ignorant vulgaire $»^{26}$, l'ouvrage s'organise autour de l'ingratitude des patients qui « meprisent le conseil du médecin $»^{27}$, et les traitements qu'il propose. C'est bien le manque de respect et de confiance pour le savoir universitaire et ses représentants que notre médecin déplore chez de tels patients.

\section{Contemptio medicinae}

Il en est tout autrement avec Les Erreurs populaires de Gaspard Bachot. Bien qu'il conserve, en continuateur, l'objectif de son prédécesseur - instruire les gens sur les abus qui se rencontraient couramment dans l'art médical - il se prononce toutefois sur un sujet voisin qui lui tient vivement à cœur, celui du mépris de la médecine. La digression occupe dès lors une place particulière dans le discours. Elle porte tout d'abord des marques très personnelles - l'auteur s'y confie et justifie son projet d'écriture ${ }^{28}$ - puis elle expose l'ensemble des raisons qui, selon Bachot, poussent ses contemporains à « mespriser la Medecine ». Ainsi, tacite dans la conception de l'ouvrage chez Laurent Joubert, la réflexion sur ce mépris dont souffrent les médecins connaît un véritable développement sous la plume de Bachot. Les sources de cette déconsidération sont au nombre de trois : " l'ignorance, la pluralité des Medecins $»^{29}$ et $«$ l'envie comme inseparable à ceux de cette profession $»^{30}$.

L'ignorance, d'abord, accompagne les médecins depuis Hippocrate. Le médecin de Cos, déjà, " se plaignoit de ce que l'ignorance de plusieurs vilipendoit la plus noble et la plus necessaire des sciences, disant que comme la timidité fait l'impuissance, l'audace engendre l'ignorance ; la science fait sçavoir, et l'opinion

\footnotetext{
${ }^{24}$ Depuis l'aube de la civilisation, la profession médicale a joui d'un prestige incontestable. Dans le discours médical, nous rencontrons souvent les vers de l'Ecclésiastique (chap. 28, 38.3), «La science du médecin lui fera tenir la teste haute, et il sera loué en présence des grands », qui deviendront topiques à cette époque. Le savoir du médecin présuppose déjà sa supériorité, renforcée par le fait qu'il tient entre ses mains la vie de ses patients. M. Koźluk, op. cit., p. 193-206.

${ }^{25}$ L. Joubert, Erreurs populaires, op. cit., $\mathrm{f}^{\mathrm{0}}$ a $6 \mathrm{v}^{\mathrm{0}}$.

${ }^{26}$ Ibid., p. 68, p. 86 ; p. 197.

${ }^{27}$ Ibid., p. 85 et p. 126.

${ }^{28}$ G. Bachot, op. cit., p. 161-162.

${ }^{29}$ Ibid., p. 164.

${ }^{30}$ Ibid.
} 
fait ignorer $»^{31}$. Mais Bachot ne s'étend pas sur ce premier motif ; il se contente d'en évoquer les origines anciennes ${ }^{32}$, le trouvant constitutif de l'histoire même de la pratique médicale.

Le second motif, la $«$ multitude des Medecins $»^{33}$, mérite aux yeux de Bachot un commentaire plus large ; il y revient deux fois au long de sa digression. Dans un premier temps, il cherche à comprendre le phénomène de la prolifération des praticiens en comparant la situation de son temps à celle de l'Antiquité. Jadis, une seule personne s'occupait des trois parties de la médecine : diététique, chirurgie et pharmacie. Ces spécialistes, rappelle-t-il, « estoient estimez plus rares ; et on les tenoit en plus grand honneur, les referant au nombre des Dieux, desquels les remedes salutaires estoient la main mesme de la divinité $\rangle^{34}$. Or, l'avènement du luxe fut aussi celui des maladies, ce qui, par conséquent, entraina la multiplication de pseudo-professionnels avides d'honneurs et de récompenses. Ces esculapes, continue Bachot, " eslevez des biens de fortune, n'ont voulu prendre la peyne d'exercer ces trois parties, tellement que les mesmes Medecins, qui avoient pour lors la faveur et l'oreille des Princes, feirent envers eux qu'ils creerent des Apotiquaires et des Chirugiens, ausquels ils monstroient la façon d'operer, comme un Architecte à ses ouvriers $»^{35}$. La séparation des compétences, souligne Bachot, marqua le moment de la division de la médecine en ses trois branches, incarnées par autant de représentants, le médecin, le chirurgien et l'apothicaire. La hiérarchie qui s'établit entre elles affirma bien évidemment, nous le savons, la prééminence du médecin. Et comme tout seigneur se nourrit de privilèges, tout vassal cherche tôt ou tard à se délivrer de la sujétion. C'est donc sans surprise, remarque Bachot, que

\begin{abstract}
ces artisans [Apothicaires et Chirurgiens] par la negligence de leurs maistres [Médecins], ont secoué de peu à peu le joug de ceste servitude, et se sont renduz si insolents (le Magistrat et le peuple qui toujours veut estre trompé y conjuant et prestant la main) qu'il y a aujourd'huy autant de Medecins que d'Apoticaires et de Chirurgiens, et qui n'implorent que l'aide des Medecins, sinon qu'à l'extremité, voire qu'il y a d'Empiriques, de Charlatans et de Triacleurs. Et que mesmes qu'on tourne indignement et contre verité, en proverbe que n'est bon Medecin qui n'est bon Charlatan. Tant il y a de corruption ès plus belles sciences ${ }^{36}$.
\end{abstract}

Son argumentation articule dans cette partie du discours la multiplication des médecins à la vénalité de leurs services professionnels - autre source du mépris qu'il déplore. Celle-ci, au départ non spécifiquement désignée par Bachot, donne lieu à une nouvelle digression enchâssée dans la principale. Chargée d'émotions

\footnotetext{
${ }^{31}$ Ibid., p. 162.

${ }^{32}$ Cf. Hippocrate, La Loi, éd. Ch. Daremberg, Paris, chez Lefèvre éditeur, 1843, p. 4-5.

${ }^{33}$ G. Bachot, op. cit., p. 162.

${ }^{34}$ Ibid., p. 163.

${ }^{35}$ Ibid.

${ }^{36}$ Ibid.
} 
et accentuée par l'évocation rhétorique du « ô tempora, o mores » cicéronien (In Catilinam I, 1.77), cette échappée marque de façon expressive ce lieu du discours bachotien :

Où sont ces anciens qui ont si souvent crié $O$ siecle, $O$ temps, $O$ mours des hommes qui vivent à présent ! que la santé des personnes si cheres à tous, un estat soit gouvernée par argent, et qu'on y introduise ceux qui en peuvent fournir, et que pour y estre en quartier et le servir, il le faille acheter ! ne se souciant celuy qui le vend de la suffisance de celuy qui l'achete, pourveu qu'il le face recevoir à sa place, et qu'il soit payé de la vente. Que ce pere de lumiere aucteur de la Medecine n'aye plus de rayons pour donner jour à la cognoissance des saints mysteres d'icelle : puis que l'avarice insatiable y a introduit ses tenebres. [...] Mais puis qu'on voit tous autres offices venaux, qui sont les plus proches de la personne du Prince, qui ne l'avoient accoustumé d'estre, il ne faut pas trouver estrange, que celuy de Medecin se vende, et aussi tost qu'un Medecin s'y perche on demande de qui et combien l'a-il acheté et ne pouvons dire autre chose, sinon, $O$ siecle, $O$ temps, $O$ meurs $!^{137}$.

Il y a des siècles déjà, Galien se plaignait de la multitude de médecins pratiquant dans la rue ; trente ans plus tôt, Laurent Joubert s'attardait sur la survivance de ce phénomène en l'illustrant brillamment d'une anecdote de Pietro Gonella, célèbre bouffon d'Alphonse I d'Este, duc de Ferrare, qui, achevant sa promenade en ville, constatait que « chacun se melle de la medecine, et y a peu de gens qui ne pansent y savoir beaucoup, voire plus que les medecins $॥{ }^{38}$. Si l'on en croit Bachot, la situation en son temps prit une telle ampleur qu'il jugea bon d'en donner à ses lecteurs un nouveau diagnostic. Nous lisons :

ceste multitude ne fust jamais plus grande qu'elle est aujourd'huy, où il n'y a si petit lieu, bourgade, ou ville, qui n'en souloit point avoir qu'y n'en aye que dix et douze, deux ou trois, d'autant que despuis qui nous avons en bon marché des lettres et en toutes Provinces des personnes qui s'y sont addonnez, qu'on a fait les cours de Theologie, Jurisprudence et Medecine tant de sortes de jeunesse de toute façon, croyant qu'ils y trouveroient leur viatique asseuré, sans considerer la difficulté et longueur des sciences qu'il ne se trouve assez de benefices pour les Theologiens, assez de causes pour les avocats, et assez de malades pour tant de Medecins ${ }^{39}$.

Bachot voit également d'un œil critique la désignation trop rapide des titres permettant aux jeunes diplômés « d'acquerir la qualité de Medecin pour se jetter promptement en quelque lieu, et y gagner de l'argent $»^{40}$. Ainsi, sans savoir ni expérience, ces nouveaux médecins partent nombreux en quête de nouveaux patients et découvrent à leur grande surprise, qu' ' il y a souvent de trop de chiens pour un os ». Un temps d'études plus long à l'université, spécule Bachot, aurait

\footnotetext{
${ }^{37}$ G. Bachot, op. cit., p. 164-165.

${ }^{38}$ L. Joubert, Erreurs populaires, op. cit., p. 95.

${ }^{39}$ G. Bachot, op. cit., p. 167-168.

${ }^{40}$ Ibid., p. 168.
} 
permis le perfectionnement de leurs connaissances, mais, hélas, « on ne les examine plus rigoureusement, $[\ldots]$ on ne leur donne pas le temps de se meurir et raffermir en toutes les parties », en conséquence de quoi « ils ne sont pas fermes et stilez encores en la profession ». En outre, ils quittent la salle « aussitost presque qu'ils entendent la langue Latine aux universitez de Medecine, d'où ils reviennent dans deux ou trois ans voire moins, jeunes et inexperts, qui est cause qu'on les mesprise ». En somme, constate Bachot, « les universitez les font naistre comme Champignons ».

L'ignorance et « l'avarice insatiable » ne contribuent certes pas à offrir une image flatteuse de la médecine. Mais Bachot ne s'arrête pas là, il allègue la troisième et dernière des causes du «mespris de ceste science $»^{41}:$ l'envie. Celle-ci est si répandue dans la profession qu'elle donna naissance, rappelle-t-il, au proverbe "Non est inuidia supra medicorum inuidiam", c'est-à-dire, il n'est plus grande envie

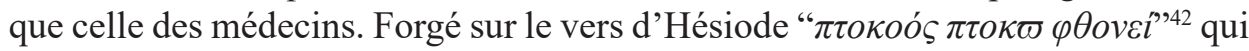
décrivaient déjà l'humanité comme naturellement envieuse depuis ses origines, le proverbe latin permet à Bachot d'insérer une véhémente diatribe contre les tristes coutumes du medicus :

l'un veut sembler tellement exceller au dessus de l'autre, qu'il mesprise tout ce qu'il fait, veut avoir la préeminence, l'honneur, le passez devant, la reputation de sçavoir sans comparaison plus que les autres, et la plus part en compagnie ou en particulier estrivent et se contrarient de tout jusques à en venir aux injures, ne disent jamais bien l'un de l'autre, taschent à la Cour des Princes, aux Univeristez, de se desnicher l'un l'autre de leurs charges et chaires, aux villes et bourgades de leurs pratiques ${ }^{43}$.

En somme, l'authentique prestige du médecin devrait être le résultat d'une " honneste emulation qui rendroit plus habille homme ou plus charitable à combattre la maladie et servir les malades ». Et bien qu'à présent « nos adversaires », explique Bachot s'en moquent et nous mettent devant nos yeux nos altercations ordinaires et nos contrariantes consultations ", la situation pourrait être renversée avec le moindre des efforts. Pour " deraciner ceste erreur », se prend-il à rêver, il suffirait « estre homme de bien, avoir la charité envers son prochain et se souvenir de ceste sentence d'Hippocrate, Que les Medecins s'assemblent et consultent pour voir ce qui est passé, présent, ou qui doibt arriver au malade, conspirant plustost au but de la santé, qu'aux altercations si frequentes, qui estant esloignées de charité, mettent l'envie, sa contraire, à sa place $»^{44}$.

\footnotetext{
${ }^{41}$ Ibid.

${ }^{42}$ Hésiode, La Théogonie, texte établi et traduit par P. Mazon, Paris, Les Belles Lettres, 1964, p. 87 : « Tout voisin envie le voisin empressé à faire fortune. Cette Lutte-là est bonne aux mortels. Le potier en veut au potier, le charpentier au charpentier, le pauvre est jaloux du pauvre et le chanteur au chanteur ».

${ }^{43}$ G. Bachot, op. cit., p. 165-166.

${ }^{44}$ Ibid., p. 166.
} 


\section{Portrait de la médecine}

L'écart entre ce vœu de Bachot et la réalité dans laquelle il vécut et exerça paraîtra bien grand. La suite de la digression dévoile en effet d'autres aspects des pratiques médicales contemporaines auxquels notre médecin s'attaque avec la même vigueur : l'ordre de préséance des médecins pendant la consultation, l'inégal prestige des facultés de médecine et la valeur de leurs diplômes, l'importance des titres permettant d'exercer les trois branches de la médecine, enfin. Voilà des problèmes délicats que Bachot se propose de commenter.

Commençons par l'acte de la consultation et ses conventions ${ }^{45}$. Celles-ci commandaient à chaque médecin invité au chevet du malade de prendre la parole pour se prononcer sur les symptômes dans un ordre défini par le rang, le titre ou l'expérience. Mais, à lire Bachot, ce parcours était surtout le théâtre de véritables discordes au cours desquelles d'arrogants médecins s'empressaient de donner leur avis devant des confrères pourtant plus diplômés, capables ainsi de témoigner à leur tour, notons-le, d'un profond mépris pour les convenances et les conventions. Dans la plupart des cas, admoneste Bachot, " il faudroit à mieux faire que les jeunes Medecins honorassent les vieux, voire s'entre-porter les uns et les autres et s'entrehonorer sans mesdire les uns des autres : qu'estans convoquez pour un malade chacun aye sa voix libre, y gardant la modestie et l'ordre requis $»^{46}$. « Nous sçavons tous le rang que nous devons tenir de la reception de nos lettres », rappelle-t-il, et il «ne faut se targuer pour le passe devant, s'entre regardant quand il faut consulter, lequel dira devant un chacun estant jaloux de cest honneur, croyant que cela serve envers le peuple à nous faire mieux appeler, si on marche devant un autre $»^{47}$. D'ailleurs, ce n'est pas sa capacité à parler avec promptitude, ou plus haut, qui fera la renommée du médecin, mais bien ses connaissances et son savoir-faire. "Croyez moy », assure Bachot, « qu'un habile homme en quelque rang qu'il marche, se trouvera tousjours habile, honoré et recherché partout $»^{48}$. Enfin, la critique bachotienne se fait plus sévère encore à l'égard des médecins de la Cour $^{49}$. Ces derniers, dénonce-t-il,

${ }^{45} \mathrm{~J}$. Coste, « Les relations entre médecins et malades dans les consultations médicales françaises (milieu XVI ${ }^{\mathrm{e}}$ siècle - début XIX ${ }^{\mathrm{e}}$ siècle) » in É. Belmas, S. Nonnis-Vigilante (éd.), Les Relations médecin-malade de la fin du Moyen Âge à l'époque contemporaine, Lille, Presses universitaires du Septentrion, 2013, URL : https://books.openedition.org/septentrion/20087; consulté le 15.12.2019.

${ }^{46}$ G. Bachot, op. cit., p. 168.

${ }^{47}$ Ibid., p. 169.

${ }^{48}$ Ibid.

49 Rappelons que « la cour est abordée en tant qu'espace de circulation privilégiée des savoirs et des hommes de savoir. Car les médecins pensionnés, du Moyen Âge jusqu'au XVIII siècle, apportent avec eux, leur formation, leur expérience ou les doctrines médicales de leur temps, toujours adaptées à leur milieu, à leur « école » et à leur clientèle. Entre Paris et Montpellier, la profession, jamais totalement unifiée, n'a cessé de décliner un éventail de savoirs, de pratiques et de discours en constante adaptation »; $\mathrm{S}$. Perrez, J. Vons, Santé et médecine à la cour de France (XVI ${ }^{\mathrm{e}}-\mathrm{XVIII}{ }^{\mathrm{e}}$ siècles), Paris, Bibliothèque interuniversitaires de santé, Collection Medic@, 2018, p. 11. 
« veulent avoir le dessus au regard des autres, bien que plus vieux, et soubs la faveur, les biens, et souvent plus la bonne fortune que la science, veulent qu'on leur cede par tout $»^{50}$. En exigeant ainsi les honneurs sans les vraiment mériter, ces médecins « font comme les Officiers de Justice, qui bien qu'ils soient bien souvent plus ignorants que les advocats tiennent neantmoins le premier rang $»^{51}$. La dignité de la profession pourrait être restaurée, termine Bachot, si les médecins renonçaient d'eux-mêmes aux disputes intestines et respectaient lors des consultations professionnelles un ordre qui reflète leurs compétences et non leur ambitions et privilèges.

La prise de parole ne devrait pas non plus être pondérée par le prestige supposé de l'université dont le médecin est issu. Bachot remarque que, bien souvent, les médecins les plus ambitieux « se targuent du lieu où ils auront pris leur Doctorat $»^{52}$. Si, à l'époque, en France, ce sont les facultés de Paris et de Montpellier qui se disputent l'excellence, il ne faut toutefois pas en déduire que les titres reçus ailleurs ont peu de valeur :

En France, Paris et Montpellier croyent que ceux qui le [le doctorat] prenent aux autres Universitez bien que privilegiées de mesme, et ayant mesme pouvoir de donner le bonnet, leur sont inferieurs : Et ceux qui le reçoivent à Bourges, Rheins, Caon, Angers, Poitiers, Cahors, Tholouse, Valence, Aix, Orenges : ne sont pas si bien docteurs qu'eux. Comme si les degrés qu'on y prend n'estoient pas esgaux aux autres ${ }^{53}$.

Bachot souligne encore tous les bénéfices qu'on trouvera à les considérer comme équivalents, d'où qu'ils proviennent :

En France tous Docteurs sont esgaux en qualité, et ne different en privileges, paissant du jour de leur reception, devant ou apres : il est encores bien mal seant de dire qu'un Docteur non seulement d'Université privilegiée, mais aussi remarquable en estude, ne puisse

Sur l'histoire curiale, voir V. Nutton (dir.), Medicine at the Courts of Europe (1500-1837), LondresNew York, Routledge, 1990 ; ou L. Brockliss, J. Colin, The Medical World of Early Modern France, Oxford, Clarendon Press, 1997.

${ }^{50}$ G. Bachot, op. cit., p. 169.

${ }^{51}$ Ibid.

${ }^{52}$ Ibid.

${ }^{53} \mathrm{Ibid} .$, p. 169-170. Plus loin dans le texte Bachot développe son propos : «Il est bien vray qu'il n y a gueres ou point d'Estude en ces lieux-là, et que pource qu'on ny va gueres pour estudier, et qu'il n'y a affluence d'Escholiers comme à Paris et Montpellier, il ne s'ensuit pas pour cela, qu'ils ayent plus de privileges que les autres, pourveu que publiquement on aye rendu tesmoignage de sa suffisance, et pris le bonnet avec l'honneur requis, apres avoir emporté le tesmoignage des docteurs soubs lesquels on a estudié aux autres Universitez de Paris ou Montpellier, ou en Allemagne, Flandres, Italie, ou Espagne, ou quelqu'autre lieu, où il y a estude et tres bon exercice de la Medecine : car en toutes Provinces il se fait d'habilles gens. Il est vray qu'il y a en chasque Royaume des privileges particuliers, et que les Cours ont jugé que les docteurs de pays estranges d'Italie comme Padoüe, Bologne, Romme, ou de Basle et autres d'Allemagne, et ainsi des autres, voire d'Avignon pour n'estre point de France, n'auroient aucun privilege en France, passent devant, mais tous autres docteurs reçeus en France jouiront / des mesmes privileges, et auront mesme ordre de leur reception », ibid., p. 170-171. 
pratiquer en une autre, sans y estre immatriculé : et qu'on se bande à poil, à crin pour cela ; et que mesmes aux grandes villes où il ny a ny estude ny Université on empesche un nouveau Docteur que s'y voudroit habituer, qu'il ny soit agregé : comme si cela estoit d'equité, pourveu qu'il ayt son doctorat et soit habille homme ${ }^{54}$.

Ces dernières réflexions visent, certes, à mettre fin à toute forme de hiérarchie favorisée par des stéréotypes séculaires, à promouvoir une confraternité structurée, mais elles permettent avant tout à Bachot de défendre ses thèses non seulement sur la préséance du médecin face à l'apothicaire et au chirurgien, mais également sur la nécessité d'une sanction de la formation de ces derniers par le médecin. « Je suis bien d'avis », dit-il, « qu'on ne reçoive en la compagnie des Docteurs Medecins, ceux qui n'ont point de grade, et qu'on ne consulte point avec eux : d'autant que cela est grandement important à la vacation, et ouvre le grand chemin aux abus qu'y s'y commettent, y introduisant les ignorants Charlatans et Empyriques et Triacleurs qui se vantent par tout d'estre Medecins $»^{55}$. Et comme tout médecin doit être approuvé par les autorités universitaires -, car «l'ordonnance du Roy defend à toutes personnes de s'inmiscuer en la pratique de la Medecine, sans estre approuvé par les Maistres de la faculté d'icelle $\aleph^{56}$, - de la même manière « les Apotiquaires et Chirurgiens doivent avoir suby l'examen de leur profession par les Medecins, comme on fait és bonnes villes, et se devroit pratiquer par tout $\aleph^{57}$. Sans ce contrôle, l'art d'Esculape perdrait sa dignité en ouvrant le chemin à l'abus et en favorisant l'ignorance.

\section{Décalogue du bon médecin}

Tout comme le bon praticien propose au malade un remède efficace pour le soulager de ses douleurs, Bachot, à la fin de sa digression, enjoint aux jeunes médecins d'adopter et de suivre un traitement éthique prescrit sous la forme d'une sorte de décalogue d'honnêtes règles professionnelles et qu'il ordonne de respecter consciencieusement :

Vous donc jeunes Medecins, Docteurs et qui avez publiquement rendu tesmoignage, en public que vous estiez capables avec le temps d'exercer ceste noble profession et vous exercer sur un si noble subjet que l'homme : craignez premierement Dieu, et songez à la creature d'iceluy que vous avec à traitter, souvenez vous de ce que Pericles disoit menant les Atheniens à la guerre. Songe à ce que tu fais, ces gens icy sont citoyens Grecs et Atheniens : aussi le corps que vous traittez c'est d'un homme caracteré à l'image de Dieu. - Ne souffrez ceux qui par l'ignorance veulent souiller vostre profession, se mettre

\footnotetext{
${ }^{54}$ Ibid., p. 171.

${ }^{55}$ Ibid., p. 171-172.

${ }^{56}$ Ibid., p. 172.

${ }^{57}$ Ibid.
} 
en vostre rang, et qu'ils soient graduez comme il faut en icelle, s'ils veulent entrer en vostre compagnie : honnorez vous les uns et les autres, les vieux les jeunes, et les jeunes respectent et honorent les vieux, les frequentent, n'ayent point d'honte d'apprendre d'eux, de les faire appeler en cas doubteux, voire leurs coetanées: Apprenez de tous ceux qui s'exercent en la profession, soit Apotiquaires, Chirurgiens, Herboristes, Operateurs, ce qu'ils sçavent bien faire, et les loüez en bien faisant. Rendez l'honneur à ceux qui vous en rendent, (et mesmes si quelque vieil Medecin n'auroit son gré, qu'il soit grandement aagé, comme il s'en trouve, pourveu qu'il soit de bonnes mœurs, sçavant, non arrogant, cedez-luy quelquesfois plus par bien-seance et par honneur de l'aage, que de droit et de devoir : ostez d'entre vous ces petites envies de vouloir paroistre plus qu'on n'est, jamais ne mesdites de vos compagnons, s'ils ne vous en ont donné subjet, et encores quand ils l'auroient fait, excusez plustost leur imbecillité que de vous vanger de leur insolence, s'il n'y a de la malice. Ne mesprisez personne de qui vous puissiez apprendre. Apprenez aux Universitez pendant que vous y avez le temps à estre bons Anatomistes, Simplistes, et voir faire les operations Chirurgicales et autres d'autant que vous ne pourriez toujours avoir ceste commodité. Ne fuyez point les Hospitaux pour y voir quantité de malades, frequentez les maistres qui meslangent bien les medicaments, exercez vous en la cognoissance des drogues, quand vous en avez la commodité, et à voir penser les blecez et autres exercices de Chirurgie ${ }^{58}$.

Si l'irritation est manifeste dans maints passages, il serait injuste, nous l'aurons compris, de voir dans la digression de Bachot la seule expression d'un ressentiment à l'égard des mœurs de profession. Il décrit, accuse, se désole et admoneste, mais propose surtout d'instruire des jeunes diplômés, comme en témoigne cette dernière exhortation, pleine de bienveillance. C'est bien l'espoir d'une profession honorable et confraternelle que Bachot exprime ici, un rêve qu'il nourrit pour l'avenir.

Cette digression de Gaspard Bachot a peut-être paru fort longue dans l'esprit de son auteur, mais sa réception quelques siècles plus tard en fait pour nous un fragment unique. Mentionnons tout d'abord que le tableau d'ensemble qu'il dresse du mépris de la médecine au XVII ${ }^{\mathrm{e}}$ siècle en France peut permettre de mieux resituer les faiblesses de l'art d'Esculape dans l'histoire de la pratique. Pour cela, il nous faudrait certes au préalable déterminer le crédit que nous pouvons accorder à Bachot en confrontant ses affirmations avec les réalités de son temps. Cependant, ce travail aurait nécessité un développement impossible à mener dans le cadre du présent article.

Il est vrai qu'il eût été intéressant d'apprécier la fidélité de l'auteur aux faits, de mettre en relief son honnêteté, de souligner, peut-être, ses exagérations, mais il n'en reste pas moins, et c'est ce sur quoi nous souhaitons insister, que le passage que nous avons analysé se définit par un profond et singulier engagement personnel.

${ }^{58}$ G. Bachot, op. cit., p. 172-174. 
À nos yeux, cette poétique de la « sincérité de l'auteur $»^{59}$ rend, en effet, la digression de Bachot particulièrement intéressante pour l'historien de la médecine. Tout comme jadis on échangeait des consultationes par lettres, dans lesquelles on n'hésitait pas à partager ses doutes sur le casus morbi ni à établir un diagnostic, Bachot, par sa posture et ses observations, instaure une relation de complicité ici inattendue, presque intime, avec son lecteur.

L'ignorance de ses confrères, leur multitude, l'avidité et les jalousies professionnelles, telles sont donc les coulisses de la contemptio medicinae, où se débattent dans la rivalité des personnages gouvernés par leurs faiblesses, guidés par leurs émotions et désireux de devancer tous les autres. Autant de raisons de mépriser cet art qui lui est cher et dont il tend à déraciner les erreurs. C'est avec vigueur qu'il s'emploie à « remuer ce tonneau Diogénique pour repurger l'auge qui salit une

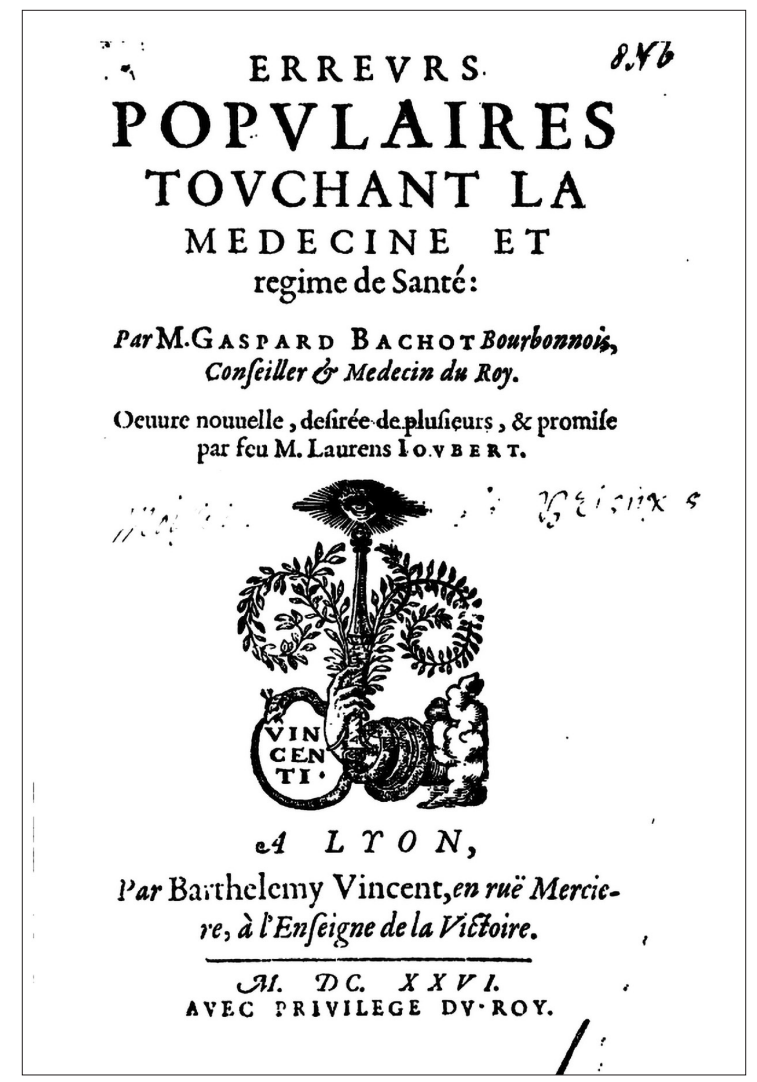

Illustration $\mathbf{n}^{\circ} 1$ : Bachot, Gaspard, Erreurs populaires touchant la médecine et régime de Santé. Euvre nouvelle, desirée de plusieurs, et promise par feu M. Laurens Joubert, Lyon, Barthelemy Vincent, 1626.

${ }^{59}$ Ibid., p. 161. 
si excellente profession $»^{60}$. En le faisant, Bachot dénonce, explique et enseigne ; en bref, il adopte une attitude didactique digne de Laurent Joubert lui-même. Il exhorte tous jeunes médecins à respecter l'ensemble des règles de comportement qui leur permettront de faire vivre la noblesse de la profession. Si seulement, achèvet-il son propos, " vous acquerez cela, vous vous empescherez que vous ne serez à mespris, d'une infinité de petits glorieux, et outrecuidez que ne peuvent à peyne bien battre au mortier ou faire une barbe $»^{61}$. Un conseil médical d'autant plus précieux qu'il ne provient pas du savoir gnomique des Anciens, mais de sa propre expérience et de sa pratique ${ }^{62}$.

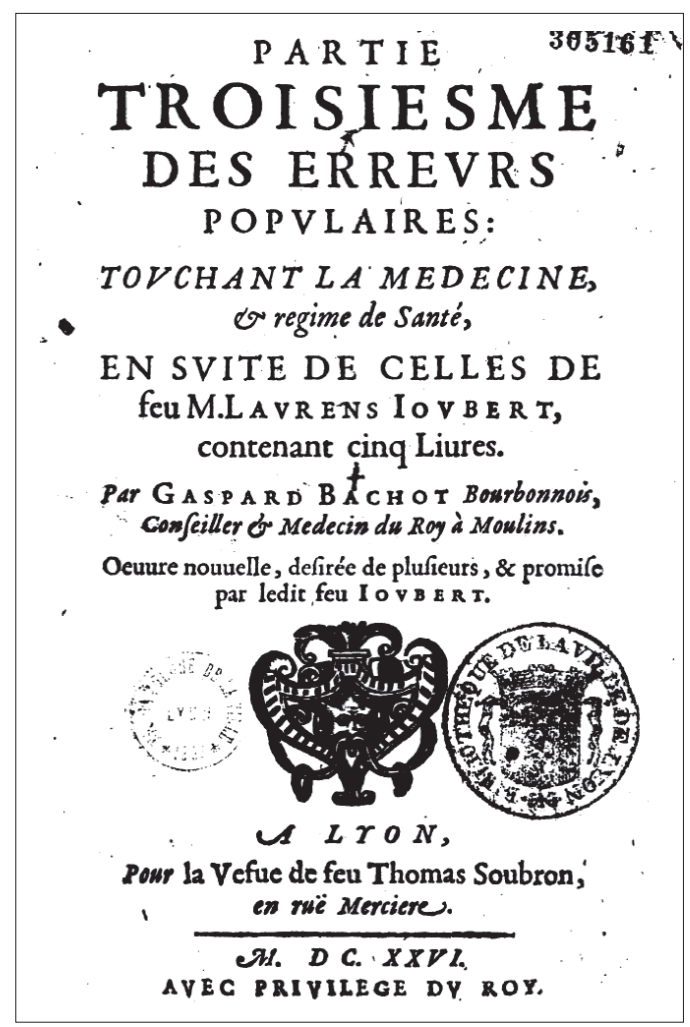

Illustration $\mathbf{n}^{\circ} 2$ : Bachot, Gaspard, Partie troisiesme des Erreurs populaires : touchant la médecine et régime de santé, en suitte de celles de feu M. Laurens Joubert, contenant cinq livres. Euvre nouvelle, desirée de plusieurs, et promise par ledit feu Joubert, Lyon, pour la veuve de feu Thomas Soubron, 1626, page de titre.

${ }^{60}$ Ibid., p. 162.

${ }^{61}$ Ibid., p. 174.

${ }^{62} \mathrm{Ibid}$. : « Je dis librement ce qui m'est arrivé commençant l'exercice jeunement à vingt trois ans : où je me suis souvent trouvé courre telles risques ». 


\section{Bibliographie}

Bachot, Gaspard, Erreurs populaires touchant la médecine et régime de Santé. Euvre nouvelle, desirée de plusieurs, et promise par feu M. Laurens Joubert, Lyon, Barthelemy Vincent, 1626

Bachot, Gaspard, Partie troisiesme des Erreurs populaires : touchant la médecine et régime de santé, en suitte de celles de feu M. Laurens Joubert, contenant cinq livres. Euvre nouvelle, desirée de plusieurs, et promise par ledit feu Joubert, Lyon, pour la veuve de feu Thomas Soubron, 1626

Berriot-Salvadore, Évelyne, Les Femmes dans la société française de la Renaissance, Genève, Droz, 1990, p. 369-390

Brockliss, Laurence, Jones, Colin, The Medical World of Early Modern France, Oxford, Clarendon Press, 1997

Broomhall, Susan, "Au-delà de la cour : patronnes et mécènes du manuscrit à l'imprimé », in Patronnes et mécènes en France à la Renaissance, Kathleen Wilson-Chevalier dir., Saint-Étienne, Publications de l'Université de Saint-Étienne, 2007, p. 45-58

Coste, Joël, La Littérature des 'erreurs populaires': une ethnographie médicale à l'époque moderne, Paris, Champion, 2002

Coste, Joël, « Les relations entre médecins et malades dans les consultations médicales françaises (milieu XVI ${ }^{\mathrm{e}}$ siècle - début XIX ${ }^{\mathrm{e}}$ siècle) », in Élisabeth Belmas, Serenella Nonnis-Vigilante (éd.), Les Relations médecin-malade de la fin du Moyen Âge à l'époque contemporaine, Lille, Presses universitaires du Septentrion, 2013, URL : https://books.openedition.org/septentrion/20087, consulté le 15.12.2019

De Rocker, Gregory, «Le rire au temps de la Renaissance : Le Traité du Ris de Laurent Joubert », Revue Belge de Philologie et d'Histoire, 1978, 56-3, p. 629-640 ; URL : https://doi.org/10.3406/ rbph.1978.3204; consulté le 16.07.2019

De Saint-Aignan, Xavier, "Les Songes de Phestion de Pierre Bailly », in Vulgariser la médecine : du style médical en France et en Italie (XVI et XVII siècles), éd. Andrea Carlino, Michel Jeanneret, Genève, Droz, 2009, p. 137-148

Dulieu, Louis, La Médecine à Montpellier, Avignon, Presses Universelles, t. II, La Renaissance, 1979

Gilles-Chikhaoui, Audrey, " Marguerite de Valois, dédicataire des Erreurs populaires de Laurent Joubert : un patronage audacieux ?» [En ligne], 1|2019, mis en ligne le 22 juin 2019, URL : http://www.siefar.org, consulté le 16.07.2019

Gorris Camos, Rosanna, « Penser le rire et rire de cœur : le Traité du ris de Laurent Joubert, médecin de l'âme et du cœur » in Rire à la Renaissance, édit. Marie-Madeleine Fontaine, Genève, Droz, 2010, p. 156-157

Hésiode, La Théogonie, texte établi et traduit par Paul Mazon, Paris, Les Belles Lettres, 1964

Hippocrate, La Loi, éd. Charles Daremberg, Paris, chez Lefèvre éditeur, 1843

Joubert, Laurent, Erreurs populaires au fait de la medecine et regime de santé, Bordeaux, Simon Millanges, 1578

Joubert, Laurent, Traité du ris, Paris, Nicolas Chesneau, 1579

Joubert, Laurent, Annotations sur toute la Chirurgie de M. Guy de Chauliac, Tournon, Claude Michel, 1598

Koźluk, Magdalena, L'Esculape et son art à la Renaissance. Le discours préfaciel dans les ouvrages français de médecine 1528-1628, Paris, Éditions Classiques Garnier, 2012, p. 193-206

Lecercle, François, Navaud Guillaume (éd). Anecdotes philosophiques et théologiques de l'Antiquité aux Lumières, Paris, Classiques Garnier, 2012

Lazard, Madeleine, «La Thérapeutique par le rire dans la médecine du XVI siècle » in Littérature et Pathologie, dir. Max Milner, Saint-Denis, Presses Universitaires de Vincennes, 1989, p. 13-27 ; URL : https://doi.org/10.4000/books.puv.1230 ; consulté le 16.07.2019

Martin-Ulrich, Claudie, La Persona de la princesse au XVI siècle : personnage littéraire et personnage, politique, Paris, H. Champion, 2004 
Michaud, Jean-François, Biographie universelle, Paris, Delagrave, 1843-1865, t. 21, p. 222.

Nutton, Vivian (dir.), Medicine at the Courts of Europe (1500-1837), Londres-New York, Routledge, 1990

Perrez, Stanis, Vons, Jacqueline, Santé et médecine à la cour de France (XVI $-\mathrm{XVIII}{ }^{\mathrm{e}}$ siècles), Paris, Bibliothèque interuniversitaires de santé, Collection Medic@,2018

Poutingon, Gérard Milhe, Poétique du digressif. La digression dans la littérature de la Renaissance, Paris, Classiques Garnier, collection «Études et essais sur la Renaissance », 2012

Tiollais, Madeleine, La Médecine et le régime de santé. Des erreurs populaires et propos vulgaires. t. I, Livre I : réfuté et expliqué par Laurent Joubert, Paris, Harmattan, 1997, p. 23-32

Magdalena Koźluk est maître de conférences à l’Université de Łódź. Elle s'intéresse aux usages de la rhétorique classique et à certains types de discours - notamment le discours médical -à la Renaissance, ainsi qu'à l'humanisme comme mode de transmission et de représentation de l'Antiquité. Outre plusieurs articles, elle a publié L'Esculape et son art à la Renaissance. Le discours préfaciel dans les ouvrages français de médecine (1528-1628), Classiques Garnier, 2012. Dans ses travaux, elle aborde la problématique du discours médical sous l'angle de l'histoire de la rhétorique, de la pensée et de l'écriture médicales comme sous celui de l'art emblématique. Orientation complémentaire de sa recherche, elle travaille également sur la bibliographie matérielle et historique appliquée aux ouvrages médicaux du XVI et du début du XVII ${ }^{\mathrm{e}}$ siècle.

\begin{tabular}{|c|c|}
\hline \multirow[t]{2}{*}{$\begin{array}{l}\text { ccreative } \\
\text { commons }\end{array}$} & $\begin{array}{l}\text { (C) by the author, licensee Łódź University - Łódź University Press, } \\
\text { Łódź, Poland. This article is an open access article distributed under } \\
\text { the terms and conditions of the Creative Commons Attribution license } \\
\text { CC-BY-NC-ND } 4.0 \text { (https://creativecommons.org/licenses/by-nc-nd/4.0/) }\end{array}$ \\
\hline & Received: 2019-01-24; Accepted: 2020-12-21 \\
\hline
\end{tabular}

